\title{
Ameloblastoma do seio maxilar: uma neoplasia odontogênica rara
}

\section{Ameloblastoma of the maxillary sinus: a rare odontogenic neoplasm}

Caio Calixto Diógenes Pinheiroํ․ Gabriela Maia Coelho². Ulisses Meireles Filgueiras Filho¹. Érika Ferreira Gomes $^{3}$. Marcos Rabelo de Freitas ${ }^{4}$. Viviane Carvalho da Silva ${ }^{5}$. André Alencar Araripe Nunes ${ }^{6}$.

1 Residente de Otorrinolaringologia, Hospital Universitário Walter Cantídio (HUWC), Universidade Federal do Ceará (UFC), Fortaleza, Ceará, Brasil. 2 Residente de Patologia, Hospital Universitário Walter Cantídio (HUWC), Universidade Federal do Ceará (UFC), Fortaleza, Ceará, Brasil. 3 Doutorado em Otorrinolaringologia, Otorrinolaringologista Assistente, Hospital Universitário Walter Cantídio (HUWC), Universidade Federal do Ceará (UFC), Fortaleza, Ceará, Brasil. 4 Doutorado em Cirurgia, Coordenador da Disciplina de Otorrinolaringologia, Universidade Federal do Ceará (UFC), Fortaleza, Ceará, Brasil. 5 Doutorado em Cirurgia, Supervisora do Programa de Residência de Otorrinolaringologia, Hospital Universitário Walter Cantídio (HUWC), Universidade Federal do Ceará (UFC), Fortaleza, Ceará, Brasil. 6 Residência Médica em Cirurgia de Cabeça e Pescoço, Chefe do Serviço de Otorrinolaringologia, Hospital Universitário Walter Cantídio (HUWC), Universidade Federal do Ceará (UFC), Fortaleza, Ceará, Brasil.

\section{RESUMO}

Ameloblastoma sinonasal é uma neoplasia odontogênica rara, cuja exérese cirúrgica com margens é o tratamento padrão-ouro. Os acessos externos tradicionais (rinotomia lateral, osteotomia Le Fort 1 e acesso transpalatal), usados isoladamente e com extensa ressecção tumoral, são associados a sérios problemas funcionais e estéticos para o paciente, afetando negativamente sua morbidade e qualidade de vida. Relata-se o caso de um homem, 68 anos, diagnosticado com ameloblastoma de seio maxilar esquerdo, cuja exérese cirúrgica foi realizada com a utilização de técnica endoscópica endonasal combinada a um acesso externo (degloving médio-facial). Após 2 anos de seguimento pós-operatório, o paciente estava sem sinais de recidiva, sem perdas funcionais e estéticas. Sugere-se o uso da técnica endoscópica endonasal, combinada ou não a um acesso externo, para reduzir a morbidade associada ao tratamento cirúrgico.

Palavras-chave: Ameloblastoma. Endoscopia. Seios paranasais.

\section{ABSTRACT}

Sinonasal ameloblastoma is a rare odontogenic neoplasm, for which the gold-standard treatment is surgical excision with margins. The traditional external accesses (lateral rhinotomy, Le Fort 1 osteotomy, and transpalatal access), used alone and with extensive tumor resection, are associated with serious functional and aesthetic problems for patients, adversely affecting morbidity and quality of life. It is reported the case of a 68 -year-old man diagnosed with ameloblastoma of the left maxillary sinus, who underwent exeresis using the endoscopic endonasal technique combined with external access (midfacial degloving). The patient has been followed up postoperatively for 2 years, with no signs of relapse, and no functional or aesthetic loss. It is suggested the use of endoscopic endonasal technique, with or without external access, to reduce the morbidity associated with the surgery.

Keywords: Ameloblastoma. Endoscopy. Paranasal sinuses.

Autor correspondente: Caio Calixto Diógenes Pinheiro, Rua Tenente Amauri Pio, 99, Meireles, Fortaleza, Ceará. CEP: $60160-090$.Telefone: +55 85 99607-8183/98966-6065. E-mail: caio_calixtodp@yahoo.com.br

Conflito de interesses: Não há qualquer conflito de interesses por parte de qualquer um dos autores.

Recebido em: 02 Fev 2018; Revisado em: 11 Mai 2018; Aceito em: 31 Mai 2018. 


\section{INTRODUÇÃO}

Com uma incidência anual de 0,5 novos casos em 1 milhão de pessoas, o ameloblastoma é considerado uma doença rara. No entanto, existem diferenças geográficas: maiores incidências são encontradas em África, China e Índia, em comparação com o mundo ocidental. ${ }^{1}$ Possui características histológicas de benignidade, raramente apresentando metástases, porém, é localmente agressivo, com altas taxas de recorrência, invasividade local e grande potencial de envolvimento de estruturas vitais. ${ }^{2}$ Cerca de $80 \%$ estão situados na mandíbula, com predileção por sua porção mais posterior. Os $20 \%$ restantes se localizam no osso maxilar, onde são particularmente perigosos, uma vez que a barreira óssea mais delgada facilita a invasão de estruturas nobres próximas, como a órbita e o crânio. A faixa etária de maior incidência varia entre 30 e 60 anos, sem predileção por sexo. ${ }^{3}$

Em 2005, a Organização Mundial de Saúde (OMS) classificou os ameloblastomas benignos em 4 subtipos: sólido/multicístico, desmoplástico, unicístico e extra-ósseo/ periférico. Atualmente, em 2017, a OMS reclassificou em 3 subtipos: ameloblastoma, ameloblastoma tipo unicístico e ameloblastoma tipo extra-ósseo/periférico. O adjetivo "sólido/multicístico" para o ameloblastoma convencional foi descartado porque não tem significado biológico e pode levar à confusão com o tipo unicístico. O subtipo "desmoplástico" deverá ser reclassificado como um subtipo histológico, pois não apresenta comportamento clinicopatológico distinto. ${ }^{4}$

O ameloblastoma maxilar possui um crescimento lento e indolor, tornando o diagnóstico difícil e tardio. Clinicamente, pode-se apresentar como sinusite, obstrução nasal, epistaxe ou assintomático. Radiologicamente, os padrões para avaliação complementar são a tomografia computadorizada e a ressonância nuclear magnética. As ameloblastomas sinonasais são, muitas vezes, indistinguíveis de pólipos nasais benignos ou de sinusite crônica, tanto clinicamente quanto na tomografia computadorizada. Embora a presença de comprometimento unilateral e de erosão óssea na tomografia computadorizada (TC) deva suscitar suspeita de uma neoplasia, a falta de características radiológicas patognomônicas exige biópsia para o diagnóstico. ${ }^{5,6}$

O padrão-ouro para seu tratamento é a excisão cirúrgica com margem óssea linear de 1 a $1,5 \mathrm{~cm} .^{5,7}$

O presente trabalho tem como objetivo compartilhar um raro caso de ameloblastoma do seio maxilar tratado cirurgicamente por meio da técnica endoscópica combinada ao degloving médio-facial.

\section{RELATO DE CASO}

Paciente masculino, 68 anos, apresentou-se no consultório com queixa de obstrução nasal e abaulamento em hemiface à esquerda há mais de 1 ano, referindo também hiposmia e episódios esporádicos de epistaxe de leve intensidade e rinorreia purulenta ipsilateral. Era hipertenso e cego à direita devido trauma ocular na infância. Exibia, ao exame físico, lesão de aspecto liso e coloração rósea em cavidade nasal esquerda, além de abaulamento do sulco gengival superior. A lesão obstruia completamente a fossa nasal esquerda, impedindo progressão da ótica de nasofibroscopia. A TC de face contrastada demonstrou formação expansiva heterogênea, captante de contraste, com epicentro no seio maxilar esquerdo, erodindo as paredes e a porção ínfero-lateral da maxila, infiltrando a gordura dos planos da face, estendendo-se para a cavidade nasal, seio frontal e células etmoidais (Figura 1).

Realizado biópsia incisional ambulatorialmente, cujo histopatológico, coloração hematoxilina e eosina (H\&E), revelou ameloblastoma sinonasal com padrão folicular predominante (Figura 2).

Figura 1. Cortes axial (à esquerda) e coronal (à direita) de TC de face contrastada evidenciando formação expansiva heterogênea, captante de contraste, com epicentro no seio maxilar esquerdo.

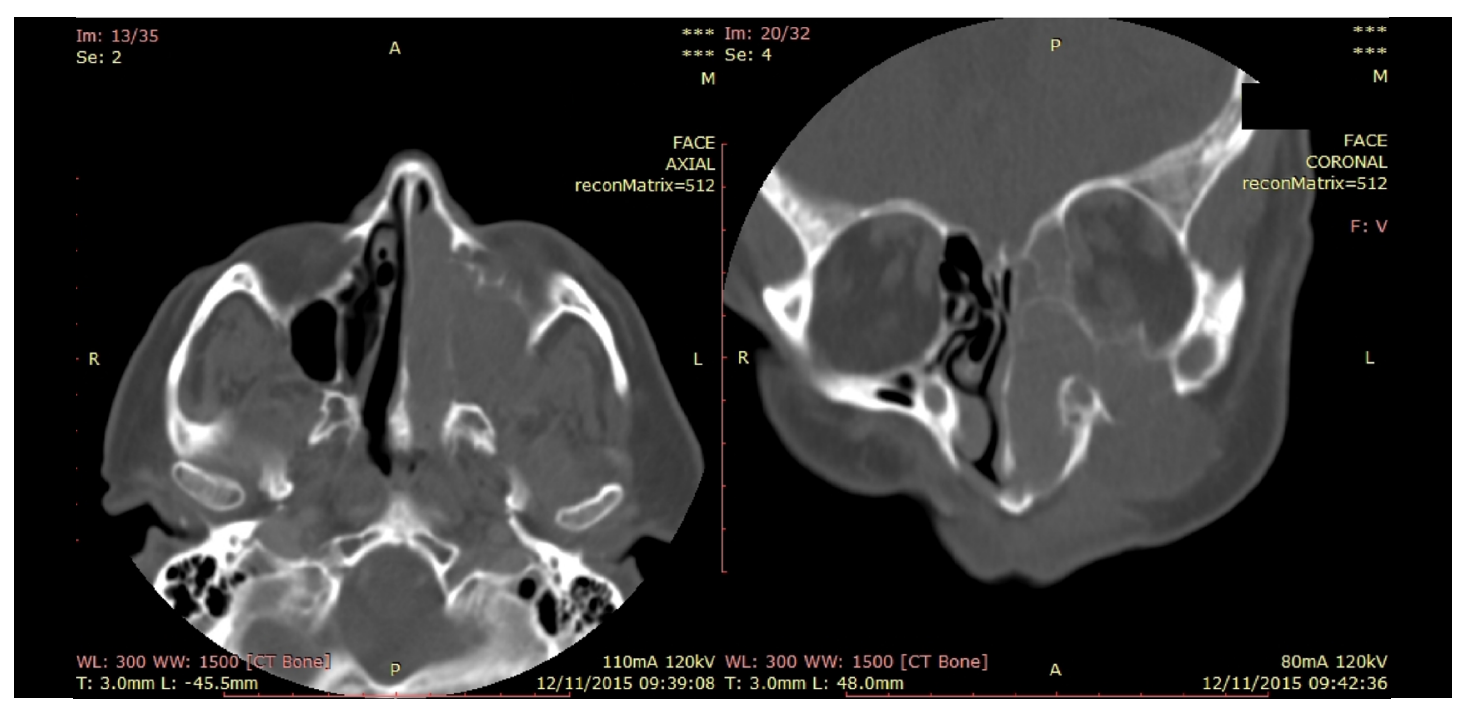


Figura 2. Histopatológico de lesão obtida pela biópsia incisional (coloração H\&E).

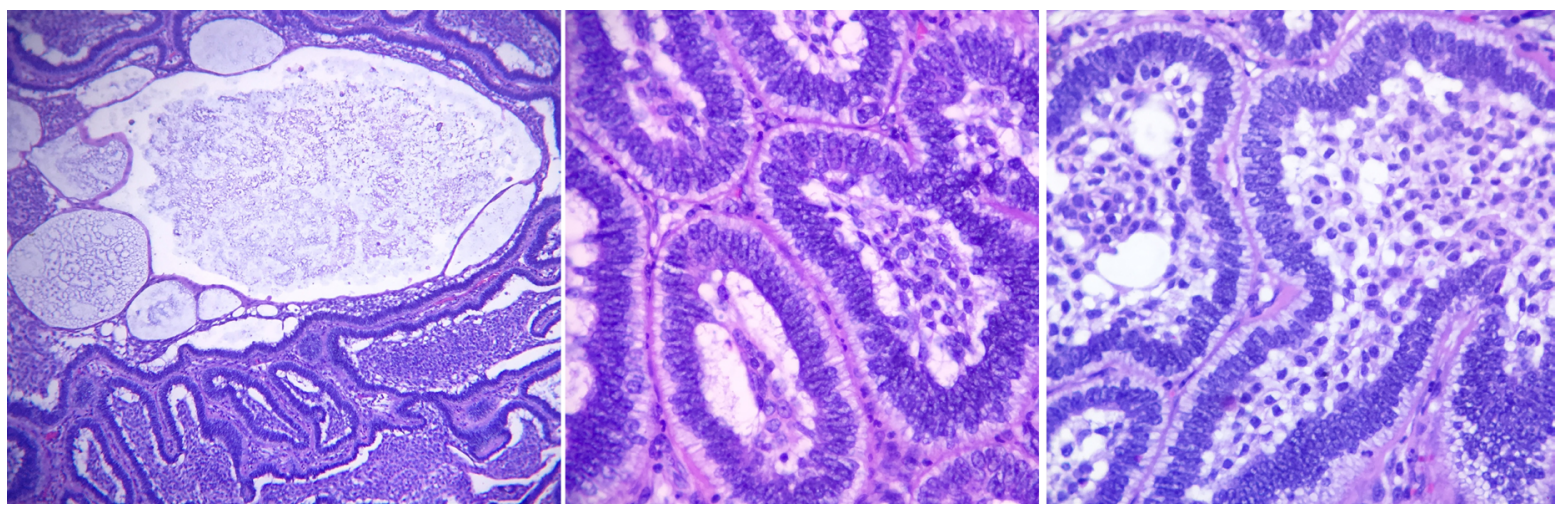

Nota: À esquerda, ilhas foliculares de epitélio odontogênico citologicamente benigno, exibindo áreas císticas (H\&E, objetiva de 10x). No centro, células colunares de característica basaloide, organizadas em paliçada, exibindo citoplasma vacuolizado e núcleos com polaridade invertida (H\&E, objetiva de 40x). À direita, estroma da lesão constituído por células frouxamente dispostas, assemelhando-se ao retículo estrelado do esmalte (H\&E, objetiva de 40x).

Foi submetido à exérese completa da lesão uma semana após resultado do histopatológico, por meio de degloving médio-facial clássico (incisões gengivobucal, septal transfixante, intercartilaginosa e na abertura piriforme) combinado ao acesso endoscópico, utilizando-se de óticas de $0^{\circ}$ e $30^{\circ}$ (Figura 3 ). No transoperatório, não foi evidenciado acometimento do assoalho da órbita, não sendo necessário sua exérese e reconstrução. Em seguimento há 2 anos sem sinais de recidiva ao exame físico e de nasofibroscopia (Figura 4).

Figura 3. Acima, imagem do transoperatório durante realização do degloving médio-facial. Imagem inferior, fossa nasal esquerda após retirada parcial da lesão e maxilectomia medial.

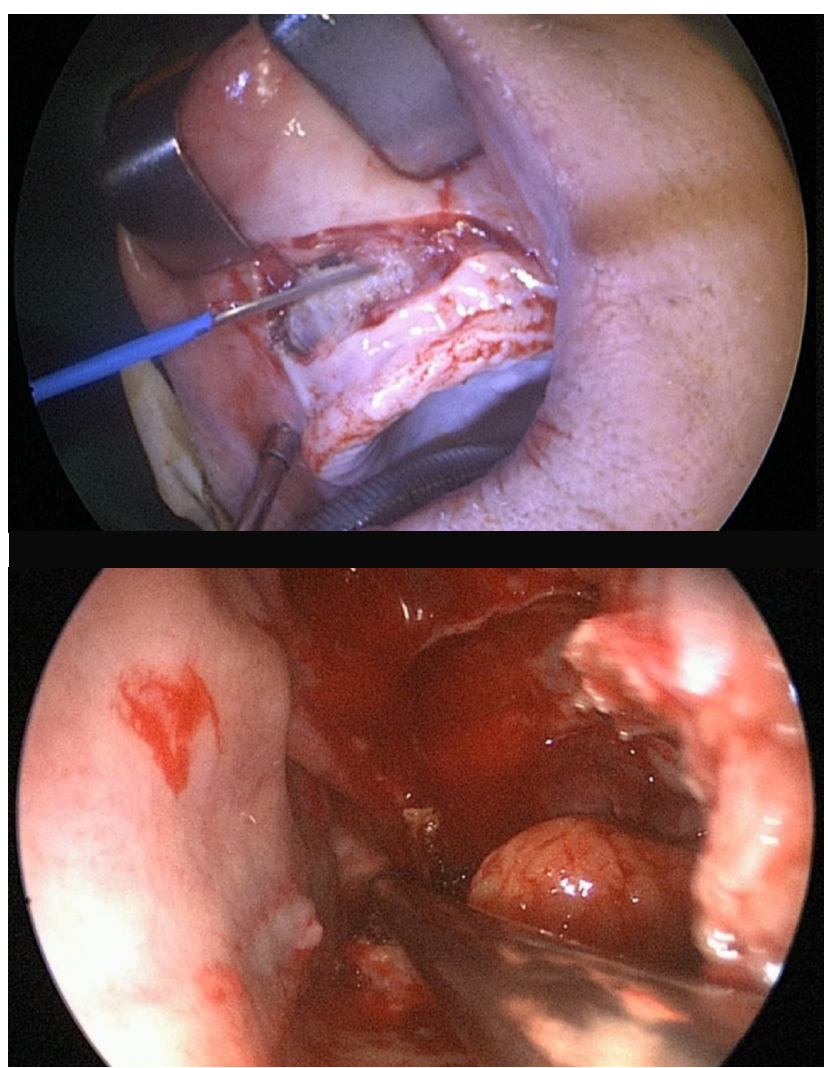

Figura 4. Nasofibroscopia de controle (um ano e seis meses após a cirurgia), mostrando ausência de lesões suspeitas. Septo à esquerda da imagem, concha média à direita. Leve edema de mucosa.

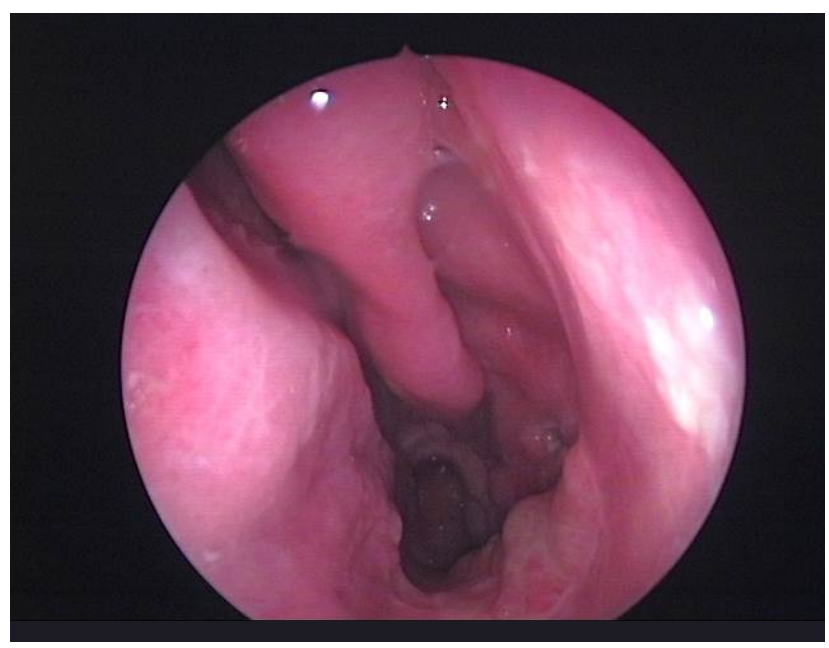

\section{DISCUSSÃO}

Como previamente exposto, a maioria dos ameloblastomas da mandíbula aparecem em pacientes de 30 a 60 anos de idade, sem predileção por sexo. ${ }^{3}$ Em contraste, os ameloblastomas dos seios paranasais têm predileção por homens com maiores idades, como em nosso caso. Alguns estudos postulam que um longo período de tempo é necessário para que os ameloblastomas sinonasais atinjam um tamanho suficiente para causar sintomatologia, sendo, portanto, diagnosticados em estágios mais avançados. ${ }^{1-3}$

O diagnóstico de ameloblastoma sinonasal é baseado na história clínica, no exame físico, na nasofibroscopia e nos exames de imagem, sendo a confirmação decisiva pelo histopatológico. Deve ser considerado no diagnóstico diferencial de tumores causando obstrução nasal: pólipo antrocoanal, papiloma invertido, carcinoma de células escamosas, adenocarcinoma, 
nasoangiofibroma, adenoma de células basais e proliferação de craniofaringioma. ${ }^{8}$

A cirurgia é o tratamento padrão para ameloblastomas. Historicamente, a extensão da ressecção tem sido controversa, composta de duas opções cirúrgicas: conservadora ou radical. A primeira envolve enucleação/curetagem da cavidade óssea, enquanto a última envolve uma operação radical com margens ampliadas (1 a 1,5cm). A enucleação simples demonstra taxas de recorrência de 60 a 90\%, sendo seu uso reservado, embora controverso, para ameloblastomas unicísticos (vistos na população pediátrica). ${ }^{7,9}$

O ameloblastoma convencional (antigamente classificado como sólido ou multicístico), tipo do caso relatado, demonstra uma maior propensão a infiltrar os tecidos circunvizinhos, apresentando, portanto, um índice mais elevado de recorrência. De maneira clássica, os ameloblastomas sólidos são tratados, na maioria dos casos, por excisões cirúrgicas radicais, que entretanto, são associadas invariavelmente a sérios problemas para o paciente, como disfunção mastigatória, mutilação, deformidade facial e movimentos anormais mandibulares, afetando negativamente a morbidade e qualidade de vida dos pacientes. $^{7,9}$

A cirurgia endoscópica tem sido amplamente utilizada, e as indicações para procedimentos endoscópicos foram estendidas a muitas doenças nasais nas últimas décadas. A abordagem endoscópica possui várias vantagens, como boa iluminação e visualização clara e ampliada, com diferentes possibilidades de angulação, resultando em cirurgia mais conservadora com dissecção precisa e menos morbidade do que a abordagem convencional. ${ }^{10}$ No entanto, em se tratando de lesões maiores, a via endoscópica exclusiva não é apropriada. ${ }^{11,12}$

\section{REFERÊNCIAS}

1. McClary AC, West RB, McClary AC, Pollack JR, Fischbein NJ, Holsinger CF, et al. Ameloblastoma: a clinical review and trends in management. Eur Arch Otorhinolaryngol. 2016;273(7):1649-61.

2. Small IA, Waldron CA. Ameloblastoma of the jaws. Oral Surg Oral Med Oral Pathol. 1955;8:281-97.

3. Dhanuthai K, Chantarangsu S, Rojanawatsirivej S, Phattarataratip E, Darling M, Jackson-Boeters L, et al. Ameloblastoma: a multicentric study. Oral Surg Oral Med Oral Pathol Oral Radiol. 2012;113(6):782-8.

4. Wright JM, Vered M. Update from the 4th edition of the World Health Organization classification of head and neck tumours: odontogenic and maxillofacial bone tumors. Head Neck Pathol. 2017;11:68-77.

5. Leong SC, Karkos PD, Krajacevic J, Islam R, Kent SE. Ameloblastoma of the sinonasal tract: a case repor. Ear Nose Throat J. 2010;89(2):70-1.

6. Jain K, Hsu J, Goyal P. The utility of a combined endoscopic and transoral resection of maxillary ameloblastoma. Int Forum Allergy Rhinol. 2013;3:762-5.
A via de acesso degloving médio-facial tem sido usada como opção para acessos externos tradicionais, como rinotomia lateral, osteotomia Le Fort 1 e acesso transpalatal, com a vantagem de evitar cicatrizes faciais e de manter exposição ampla bilateral da cavidade nasal. ${ }^{11}$ A técnica endoscópica endonasal, combinada ou não ao degloving médio-facial, reduz o tempo transoperatório, a morbidade e o tempo de hospitalização, mantendo a mesma efetividade que as cirurgias abertas tradicionais (rinotomia lateral, osteotomia Le Fort 1 e acesso transpalatal) para tratamento de tumores nasossinusais (como o ameloblastoma, o papiloma invertido e o tumor odontogênico queratocístico). ${ }^{6,10-15}$

A taxa de recorrência é um fator crucial para um planejamento cirúrgico coerente, entretanto, outros aspectos são também relevantes e devem ser levados em consideração no momento da proposta terapêutica, destacando-se a morbidade e qualidade de vida dos pacientes. ${ }^{9}$

É importante salientar que, independentemente da técnica utilizada, é imprescindível um longo controle clínico-radiográfico dessas lesões, já que, apesar de mais de $50 \%$ das recorrências ocorrerem até 5 anos após a cirurgia, há casos de até 10 a 15 anos depois. ${ }^{11}$

\section{CONCLUSÃO}

O ameloblastoma maxilar possui difícil tratamento, principalmente em comparação com seu correspondente mandibular, devido, sobretudo, à maior proximidade e ao risco de invasão de estruturas nobres, como órbita, cavidade nasal e base do crânio. A técnica endoscópica, entretanto, pode minimizar a morbidade associada com o tratamento cirúrgico.

7. Carlson ER, Marx RE. The ameloblastoma: primary, curative surgical management. J Oral Maxillofac Surg. 2006;64(3):484-94.

8. Tranchina MG, Amico P, Galia A, Emmanuele C, Saita V, Fraggetta F. Ameloblastoma of the sinonasal tract: report of a case with clinicopathologic considerations. Case Rep Pathol. 2012;2012:218156

9. McClary AC, West RB, McClary AC, Pollack JR, Fischbein NJ, Holsinger CF, et al. Ameloblastoma: a clinical review and trends in management. Eur Arch Otorhinolaryngol. 2016;273(7):164961 .

10. Mun MJ, Jung DW, Lee CH, Cho KS. Endoscopic removal of a huge keratocystic odontogenic tumor in maxillary sinus. J Craniofac Surg. 2014;25(2):586-8.

11. Ferreira LM, Rios AS, Gomes EF, Azevedo JF, Araújo RP, Moraes RB. Degloving médio-facial como via de acesso a tumores nasossinusais. Rev Bras Otorrinolaringol. 2006;72(2):158-62.

12. Larget I, Bastier PL, Gabory L. External versus endoscopic approach in the management of 131 sinonasal inverted papillomas. Rev Laryngol Otol Rhinol (Bord). 2015;136(1):3-7. 
13. London SD, Schlosser RJ, Gross CW. Endoscopic management of benign sinonasal tumors: a decade of experience. Am J Rhinol. $2002 ; 16: 221-7$.

14. Goudakos JK, Blioskas S, Nikolaou A, Vlachtsis K, Karkos P, Markou KD. Endoscopic Resection of Sinonasal Inverted Papillomas: Systematic Review and Meta-Analysis. Am J Rhinol Allergy. 2018;32(3):167-74.
15. Kılıç S, K1lıç SS, Baredes S, Chan Woo Park R, Mahmoud O, Suh $\mathrm{JD}$, et al. Comparison of endoscopic and open resection of sinonasal squamous cell carcinoma: a propensity score-matched analysis of 652 patients. Int Forum Allergy Rhinol. 2018;8(3):421-34.

\section{Como citar:}

Pinheiro CC, Coelho GM, Filgueiras UM Filho, Gomes EF, Freitas MR, Silva VC, et al. Ameloblastoma do seio maxilar: uma neoplasia odontogênica rara. Rev Med UFC. 2018 out-dez;58(4):66-70. 